\title{
Performance Characterization of IEEE 802.11s Compatible MAC Protocols
}

\author{
A.B Nataraju, S.A Hariprasad
}

\begin{abstract}
Wireless Mesh Network (WMN) [1] deployment based on IEEE 802.11s [2] standard is a popular choice in setting up cost-efficient alternative to support broadband internet services to a larger population. They can provide network connectivity over large geography when compared to WLANs. WMN makes use of Distributed Coordination Function (DCF) Medium Access Control (MAC) protocol with Binary Exponential Backoff (BEB) algorithm to avoid collision due to simultaneous transmissions by more than one user at the same time. These collisions bring the network performance drastically down if not handled properly. Thus this work tries to evaluate the suitability of the better efficient CA algorithms (EIED, EILD, MILD, PB, CCW, EBO, $H B A$...) which can replace $B E B$ to support higher throughput for IEEE 802.11s networks (WMNs). The NS-3 [3] simulation results indicate that Polynomial Backoff (PB) performs better than others against the BEB algorithm in about $52 \%$ of network scenarios and it is suggested to operate with TWO radios enabled with all access points.
\end{abstract}

Keywords: IEEE 802.11s, WMN, BEB, MAC, HWMP, MRMC

\section{INTRODUCTION}

$W_{\text {MNs have been projected as the most preferred }}$ alternative for next-generation wireless networks with least cost last-mile connectivity. WMNs essentially make use of multi-hop communication to support wireless services over a large area. At the heart, the Mesh Router provides internet access to the Mesh Clients. A Mesh Router can be built out of general-purpose computing devices like, laptops, desktops, or on dedicated systems. The simplicity in adding the new routers make WMNs as the preferred technology for applications viz., Advanced Metering Infrastructure (AMI), intrusion detection systems, remote video surveillance, smart grids, environmental monitoring. In many applications, WMNs are expected to support internet services to heterogeneous clients over a large area.

Figure 1. Depicts the need to adopt multi radio multi channel features on Mesh Router to achieve maximum throughput, but is not required for mesh clients to achieve the best results from WMNs.

Revised Manuscript Received on February 05, 2020.

* Correspondence Author

Nataraju A.B*. Assistant Professor, Dept. of ECE, Acharya Inst. Of Technology, Research Scholar, Jain University, Bangalore, INDIA nataraju.ab@gmail.com

Hariprasad S.A, Director, School of Engineering and Technology, Jain University, Bangalore, INDIA hariprasad.sa@jainuniversity.ac.in

(C) The Authors. Published by Blue Eyes Intelligence Engineering and Sciences Publication (BEIESP). This is an open access article under the CC BY-NC-ND license (http://creativecommons.org/licenses/by-nc-nd/4.0/)
The WLAN standard was originally designed to support best-effort services, in providing high throughput and fairness in resource allocation.

Increased demand for wireless Internet access has led to rapid growth in WLANs in recent years. The lion's share of this burgeoning market has been captured by products based on the IEEE 802.11 family of standards. Most of the traffic carried on a typical WLAN is made up of non-real-time applications such as web browsing and email. WMN is advancement over WLAN, which can provide wireless connectivity over a relatively large geography when compared to WLAN. WMNs are undergoing rapid progress

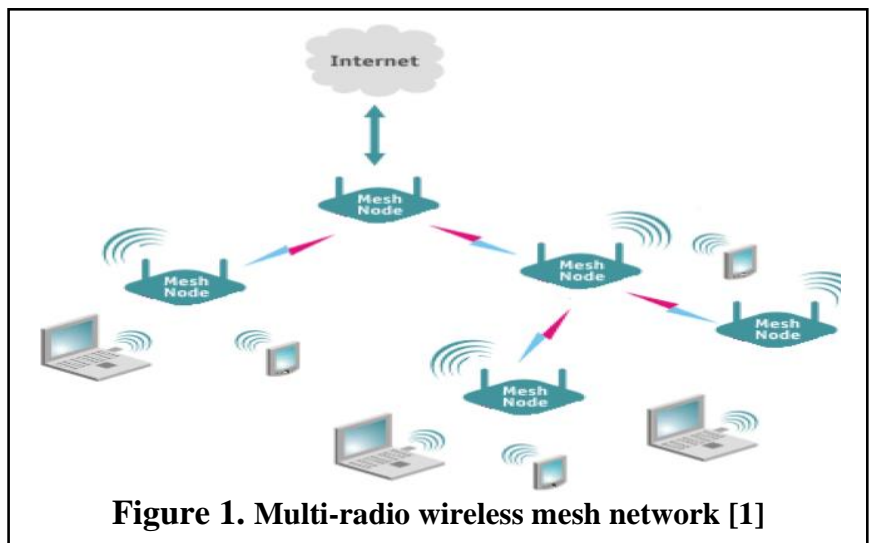

and inspiring numerous applications due to their enhanced capability against WLANs. However, many technical issues still exist in WMNs.

Some of the common sets of problem areas, which needs to be handled in order to achieve better WMN performance are (a) choosing the most efficient backoff algorithm which has been developed for WLAN instead of the default BEB algorithm, (b) choosing the most efficient routing protocol which can work with complex meshed connections which makes wireless connectivity more and more complex to manage and support user data transmission, (c) multi radio devices can support better throughput but there is not much literature has been published on multi radio backoff schemes, which needs a serious deliberation on suitability of multi radio (MR) backoff (BO) schemes instead of single radio BO schemes. Randomized BEB plays a pivotal role in coordinating medium access by multiple devices to a shared communication medium. On the contrary BEB is expected to underperform, when the traffic is "bursty". Also there are huge effort has gone into designing newer back off $\mathrm{BO}$ algorithms for various wireless standards. There raises a question, Is BEB still a best choice for wireless networks. In [4] authors have inferred that newer BO algorithms significantly underperform in many network setups. 


\section{Performance Characterization of IEEE 802.11s Compatible MAC Protocols}

They have also inferred that number of collisions is not the only root cause for underperformance. Thus this work tries to evaluate the BEB's suitability for WMN setups.

The main practical problem of random multiple accesses in a wireless channel is the collision of transmitted packets.

The collisions can degrade the throughput and fairness efficiency of WLAN / WMN. Backoff algorithm is an important component which can be enhanced to reduce the collision probability. One of the simplest ways to reduce the collision problem is the usage of a sampling random-time to delay the next retransmission packet. Significantly, a key concept of designing back-off algorithms is how to select an optimum contention window size for the maximum throughput, fairness index and smallest packet delay.

\section{RELATED WORK}

Substantial research has gone into evaluating the performance of WMNs with different network densities (grid sizes). In [5] , authors have evaluated the performance of 802.11s in comparison with $802.11 \mathrm{~g} / \mathrm{b} / \mathrm{a}$ type of nodes. They have found that multi-hop communication substantially reduces the performance of IEEE 802.11n. They have also found that WMN with layer-3 routing (ex. AODV) results in better performance when compared to layer-2 routing protocols (ex. HWMP, PMP). Also they have observed that the 802.11n with multi-hop communication perform poorly when compared to single-hop communication. This necessitates identification of a backoff scheme instead of BEB which works well with WMN implementations.

$\mathrm{BEB}$ is the earliest MAC protocol used in CSMA protocols to reschedule packets after a collision. In BEB, a node attempts to transmit a packet following a backoff interval that is randomly selected from a backoff window; in response to a collision the window is multiplied by a factor of TWO in order to reduce the probability of collision during the next transmission. There are quite a few enhancements proposed in the literature viz., EIED, EILD, DIDD, MILD, QB, LB mechanisms (broadly classified as exponential, linear and polynomial backoff schemes). But there is not much work has been published specifically suited for wireless mesh networks (WMN). In this work we propose an analysis of backoff algorithms suitable for WMNs. In this work we assume WMN setup based on IEEE 802.11s standard, which in turn uses HWMP and PMP protocols for link scheduling and routing operations.

\section{Linear backoff scheme [6]}

$W_{i}= \begin{cases}\left\langle(\beta i+1) W_{0}\right\rangle, & \text { for } i=0, \ldots, m-1, \\ \left\langle(\beta m+1) W_{0}\right\rangle . & \text { for } i=m, \ldots, R-1,\end{cases}$

Exponential backoff scheme [6]

$W_{i}= \begin{cases}\left\langle\beta^{i} W_{0}\right\rangle, & \text { for } i=0, \ldots, m-1, \\ \left\langle\beta^{m} W_{0}\right\rangle . & \text { for } i=m, \ldots, R-1,\end{cases}$

Polynomial backoff scheme [6]

$$
W_{i}= \begin{cases}\left\langle(i+1)^{\beta} W_{0}\right\rangle, & \text { for } i=0, \ldots, m-1, \\ \left\langle(m+1)^{\beta} W_{0}\right\rangle . & \text { for } i=m, \ldots, R-1 .\end{cases}
$$

Jesada Sartthong et.al, [7] proposed a new backoff algorithm named half binary exponential Increase and Double Decrement (HBEIDD) which perform better throughput than BEB, EIED [8], DIDD [9], BEIHD [10], RIBED under heavily loaded WLANs. But there is no guarantee on its suitability for WMNs.

Zygmunt J. Haas et.al, [11] have found that the optimum BO interval should be FOUR times the transmission time of a packet when the random access channel operates under a pure ALOHA scheme. They have observed that the BO algorithms which select the CW based on the node density can perform better than BEB in WLAN setup

On the same lines, Nataraju A.B. et.al, [12] observed that the network performance can be enhanced if the medium access protocol based its operation on network node density.

Sakurai et.al, [13] observed that the BEB mechanism induces a heavy-tailed delay distribution in case of unlimited retransmissions. Also they have inferred that DCF is prone to long delays and not suited to carry delay-sensitive data.

Anderton et.al, [4] have observed BEB may not be a best medium allocation algorithm under many of the application scenarios. Thus the work carried out in this work finds a greater significance for WMN throughput enhancement.

B. Nithya et.al, [14] proposed an Integer Sequences based Backoff Algorithm (ISBA) which exploits cubic, exponential, jacobsthal and catalan integer sequences to estimate the proper Contention Window (CW) size. Based upon backoff stages and failure count of acknowledgments, these integer sequences are used to accomplish the adequate growth rate of $\mathrm{CW}$. This leads to relatively efficient medium access to curtail end-to-end delay and collisions among contending stations.

Srikrishna Sridhar et.al, [15] have proposed a channel to radio mapping scheme in MRMC based WMNs. They have constructed a model for channel assignment as an optimization problem with the goal of minimizing the overall network interference. The problem has been proven to be NP-Hard. Through extensive simulations they have demonstrated that the distributed algorithm performs competitively and can serve as a practical and scalable solution to the channel assignment problem.

Xinghua Sun et.al, [16] observed that the BEB may not provide best performance in WLAN environment when compared to other backoff algorithms. They have also observed that BEB can achieve the theoretical limit of throughput when the initial backoff window size is properly selected. It, however, suffers from significant delay degradation when the network becomes saturated. They have also inferred that polynomial backoff support better throughput compared to exponential backoff schemes. They have also inferred that the polynomial backoff with degree $(\mathrm{x}=2)$ support the better performance when compared with higher order polynomial, $x=3,4,5$.

Suzhi et.al, [17] have observed that the BEB mechanism, the key collision avoidance scheme in DCF of 802.11, is fundamentally defected in inducing divergent moments of medium access delay. The delay variance can easily approach infinity with pragmatic system configurations, which translates into service starvation for some users and eventually leads to severe service inequality among users. 
The authors have shown that the application of power law delay can be mitigated by swapping BEB with polynomial back-off (PB). They have found, through a rigorous analysis, that all delay moments are finite with polynomial backoff, and thus fundamentally fix the problem of starvation and inequality. In addition, PB yields higher throughput with a practical network size when the order of the polynomial backoff function is set reasonably

Balador et.al, [18] inferred that the network performance can be improved when the multiple channels are used simultaneously over multi radios using different frequency bands. But for this mechanism to work properly the channels shall be separated spatially far apart to avoid any interference to communications over other channels at the same time. It is also observed the channels $(1,6,11$, and 14$)$ or $(2,7,12)$ or $(3,8,13)$ can be used by routers / nodes simultaneously over different logical or physical radios. Supporting multiple simultaneous communications over channels separated in frequency domain is called as multi-radio / multi-interface based communication. The multi radio feature can supported over existing $802.11 \mathrm{~b} / \mathrm{g} / \mathrm{n}$ hardware with software update or with brand new hardware to support the multi-radio functionality over multiple antennas.

Ruopeng Wang et.al, [19] proposed a modification to DCF with constant contention window which is dependent upon scale of network to obtain the maximum throughput where the user density is fixed for a given application scenario.

In [20], the authors have proposed an Enhanced BO (EBO) algorithm with different contention intervals dependent upon back off stage. The proposed hybrid algorithm is dynamic in nature and found to absorb the collisions more efficiently than BEB and other older variants of backoff algorithms.

\section{PROBLEM FORMULATION \& METHODOLOGY USED}

The main goal of this work is to identify the optimal number of radio interfaces, packet size; and backoff algorithm to achieve peak throughput performance for WMNs.

\footnotetext{
Methodology for performance characterization

Initialization:

gridSize $=3 \times 3,4 \times 4,5 \times 5,6 \times 6,7 \times 7$;

nInternfaces $=1,2,3$;

packetSize $=400,600,800,1000,1200,1400,1500,1600,1800,2000$;

RngRun = 11, 22, 33, 44, 55, 66, 77, 88, 99, 101, 111, 122, 133, 144, 155 166, 177, 199, 201, 211; // 20 values

Methodology:

1: Repeat for all gridSize, // realized by m_xSize \& m_ySize

2: Repeat for all nInternfaces, // realized by m nIfaces

3: $\quad$ Repeat for all packetSize // realized by m_packetSize

4: $\quad$ Repeat NS-3 simulation with different RngRun

5: $\quad$ Run the NS-3 simulation with specific gridSize, packetSize interfaces, and Random Seed number(RngRun)

. / waf --command-template $=" \% \mathrm{~s}$

$--m \_x$ Size=3 - -m_ySize=3

$--m$ packetSize $=400 \quad-m$ nIfaces $=1$

$--m$ step $=170 \quad--$ RngRun=11" - -run

scrātch/HwmpGrid

7 :

$\rightarrow$ This statement simulate $3 \times 3$ grid, nInterfaces $=1$,

PacketSize = 400;

Save the results; // $\rightarrow$ result-1, 2, 3,...20

End - Repeat NS-3 simulation

Find the median of these 20 iterations and tabulate for

analysis/characterization.

End - Repeat for all packetSize

End-Repeat for all nInternfaces

End - Repeat for all gridSize

14: Repeat the above steps 1-13 for different MAC CA algorithms viz., $B E B, D I D D, E I E D, E B O, H B O, P B, C C W, \ldots$
}

15: Compare the Throughput from BEB with other algorithms. The differential values are tabulated in Table 2 - Table 11.

For instance, Table 2 : indicates difference in Throughput from EIED and BEB algorithms $\rightarrow$ THEIED - TH

Similarly, Table 3 : indicates difference in Throughput from EIED and BEB algorithms $\rightarrow \mathrm{TH}_{\mathrm{DIDD}}-\mathrm{TH}_{\mathrm{BEB}}$.

Continue similarly for other CA algorithms as well for performance characterization.

The following section discuss the experimental results obtained using the methodology mentioned and inferences drawn accordingly.

\section{EXPERIMENTAL SETUP FOR CONTENTION AVOIDANCE ALGORITHM TEST}

The following results have been observed from the extensive simulations using NS-3 simulator and the inferences have been drawn accordingly. The set of configurations considered in these simulations are listed in

Table 1.

Table 1- Simulation parameters [21]

\begin{tabular}{|c|c|}
\hline Parameter & Values \\
\hline $\begin{array}{l}\text { Computing } \\
\text { environment and } \\
\text { Operating System }\end{array}$ & $\begin{array}{l}\text { Ubuntu } 16.04 \text { LTS } \\
\text { HP Compaq } 8200 \text { Elite MicroTower - } \\
\text { 4GM RAM, 500GB HDD } \\
\text { Core-i5, 3.09GHz processor. }\end{array}$ \\
\hline Grid size $(P \times Q)$ & $3 \times 3,4 \times 4,5 \times 5,6 \times 6,7 \times 7$ \\
\hline Step size (metre) & 170 \\
\hline $\begin{array}{l}\text { Radio Propagation } \\
\text { Model }\end{array}$ & $\begin{array}{l}\text { ns3::ConstantSpeedPropagationDelayM } \\
\text { odel }\end{array}$ \\
\hline $\begin{array}{l}\text { Propagation Loss } \\
\text { Model }\end{array}$ & $\begin{array}{l}\text { ns3::LogDistancePropagationLossMode } \\
\text { l }\end{array}$ \\
\hline Payload size (bytes) & $0,4,0.6,0.8, \ldots 2.0 \mathrm{~KB}$ \\
\hline Simulation time (sec) & 175 \\
\hline $\begin{array}{l}\text { No of simulation } \\
\text { scenarios (driven by } \\
\text { different random } \\
\text { seed numbers, } \\
\text { RngRun } \\
=11,22,33,44,55 \text {, } \\
66,77,88,99,101 \text {, } \\
111,122,133,144, \\
155,166,177,188, \\
199,211 \text { ) }\end{array}$ & 20 \\
\hline Topology & Grid \\
\hline $\begin{array}{l}\text { Routing protocols } \\
\text { considered }\end{array}$ & HWMP+PMP (IEEE 802.11s) \\
\hline $\begin{array}{l}\text { Number of radio } \\
\text { interfaces (channel } \\
\text { no. }-0,5,10)\end{array}$ & $1 / 2 / 3$ \\
\hline $\begin{array}{l}\text { Number of nodes }= \\
\text { MxN }\end{array}$ & $9 / 16 / 25 / 36 / 49$ \\
\hline $\begin{array}{l}\text { No. of Connections / } \\
\text { flows }\end{array}$ & $9 / 16 / 25 / 36 / 49$ \\
\hline $\begin{array}{l}\text { EnergyDetectionThre } \\
\text { shold }\end{array}$ & $-89.0 \mathrm{dbm}$ \\
\hline CcaMode1Threshold & $-62.0 \mathrm{dbm}$ \\
\hline WifiPhyStandard & WIFI_PHY_STANDARD_80211b \\
\hline RtsCtsThreshold & 2200 (Disabled) \\
\hline User application & ns3::OnOffApplication \\
\hline Application data rate & 150kbps \\
\hline
\end{tabular}




\section{Performance Characterization of IEEE 802.11s Compatible MAC Protocols}

Channel allocation

schemes considered.

BEB, EIED, EILD, DIDD, PB(2), CCW, EBO, HBO

HWMP protocol performance has been analyzed against the system Throughput parameter, which is defined as:

Throughput (bps): it is a measure of number of application data bytes received by the receiver in one unit of time.

$$
\underline{\text { Throughput }}=\frac{\sum \text { Total no. of app data bytes received }}{\sum \text { Total simulation time }}
$$

Contention window ranges for different MAC protocols are set as follows.

\section{1) BEB - Binary Exponential Backoff [22]}

The contention window range is doubled after each failure and reset to minimum value upon successful transmission. $C W=\left\{\begin{array}{lr}\min (2 * C W, C W \max ) & \text { on collision } \\ C W \min & \text { on success }\end{array}\right\}$

Backoff range $\{0, C W\}$

Backoff Timer $\leftarrow$ Rand $(0, C W) *$ slotTime;

\section{2) EIED - Exponential Increase Exponential Decrease}

The contention window range is doubled after each failure and reset by a factor of $\sqrt{2}$ upon successful transmission [8].

$$
C W=\left\{\begin{array}{ll}
\min (2 * C W, C W \max ) & \text { on collision } \\
\max \left(\frac{C W}{\sqrt{2}}, C W \min \right) & \text { on success }
\end{array}\right\}
$$

Backoff range $\{0, C W\}$

Backoff Timer $\leftarrow$ Rand $(0, C W) *$ slotTime;

3) DIDD - Double Increase Double Decrease [9]

$C W=\left\{\begin{array}{ll}\min (2 * C W, C W \max ) & \text { on collision } \\ \max (C W / 2, C W \min ) & \text { on success }\end{array}\right\}$

Backoff range $\{0, C W\}$

Backoff Timer $\leftarrow$ Rand $(0, C W) *$ slotTime;

\section{4) MILD - Multiplicative Increase and Linear Decrease} $C W=\left\{\begin{array}{lr}\min (C W * 1.5, C W \max ) & \text { on collision } \\ \max (C W-x, C W \min ) & \text { on success }\end{array}\right\}$

Backoff range $\{0, C W\}$, and $x=32$.

Backoff Timer $\leftarrow$ Rand $(0, C W) *$ slotTime; [22]

5) EILD - Exponential Increase Linear Decrease [22] $C W=\left\{\begin{array}{ll}\min (2 * C W, C W \max ) & \text { on collision } \\ \max (C W-x, C W \min ) & \text { on success }\end{array}\right\}$

Backoff range $\{0, C W\}, x=32$ or 64 .

Backoff Timer $\leftarrow$ Rand $(0, C W) *$ slotTime;

6) EBO - Enhanced Backoff algorithm [23] slot_lb $[\mathrm{N}]=\{0,32,96,224,480,992\}$

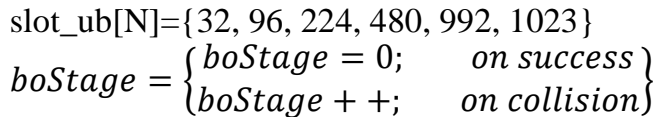

Backoff range \{ slot_lb[boStage], slot_ub[boStage] \}

Backoff Timer $\leftarrow$ Rand (slot_lb[boStage], slot_ub[boStage]) * slotTime;

\section{7) PB - Polynomial Backoff (PB_PAPER_2) [17]}

$\beta=2$;

boStage $=\left\{\begin{array}{l}\text { boStage }=0 ; \quad \text { on success } \\ \text { boStage }++; \quad \text { on collision }\end{array}\right\}$
$C W_{\text {bostage }}=(1+\beta)$ boStage $* W_{0}$
Backoff range $\left\{0, C W_{\text {bostage }}\right\}$
Backoff Timer $\leftarrow$ Rand $\left(0, C W_{\text {bostage }}\right) *$ slotTime;

8) HBO - Hybrid BackOff algorithm [20]

$\mathrm{m} 1=2, \mathrm{~m} 2=8, \mathrm{a}=240, \mathrm{~W}_{0}=\mathrm{cmMin}(31)$

boStage $=\left\{\begin{array}{lc}\text { boStage }=0 ; & \text { on success } \\ \text { boStage }++; & \text { on collision }\end{array}\right\}$

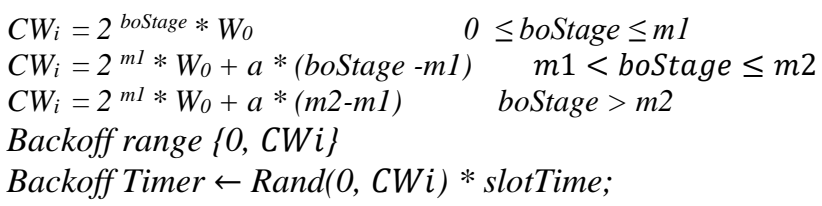

9) CCW -Constant Contention Window algorithm [19]

Contention window remains same irrespective of success or failure in communication $\left(\mathrm{CW}_{\text {fixed }}=300\right.$ or 400$)$.

$\mathrm{CW}=\left\{\begin{array}{lc}\text { CWfixed } & \text { on collision } \\ \text { CWfixed } & \text { on success }\end{array}\right\}$

Backoff range $\{0, C W\}$

Backoff Timer $\leftarrow$ Rand $(0, C W) *$ slotTime;

\section{Results Analysis \& Discussion}

In this section, we present the results obtained and inferences are drawn accordingly.

Note for decoding labels used in Table 2 - Table 11

$3 x 3 \_2 \rightarrow$ grid size $=3 \times 3$, number of Interfaces $=2$

$7 x 7 \_3 \rightarrow$ grid size $=7 x 7$, number of Interfaces $=3 \ldots$

SIZE $\rightarrow$ Packet SIZE

Each cell value indicates Throughput with row indicating packet size and column indicating node density, \& No. of interfaces enabled with mesh router.

A cell in the table with green/gray shade indicates BEB underperform when compared to other MAC protocol. Table 2 - Table 11, list out the difference in throughput from two different CA algorithms.

For instance, when $\mathbf{T H}_{(\mathbf{E I E D} 3 \mathbf{3} 3 \text { _i1) }}>\mathbf{T H}_{\left(\mathbf{B E B} 3 \mathbf{3} 3 \_ \text {_i1) }\right.}$ leads to positive value (gray shaded cell).

\begin{tabular}{|c|c|c|c|c|c|c|c|c|c|c|c|c|c|c|c|}
\hline \multicolumn{16}{|c|}{ THEIED - TH BEB } \\
\hline SIZE & 3x3_1 & $3 \times 3$ & $3 \times 3 \_3$ & $4 \times 4$ & $4 \times 4 \_2$ & $4 \times 4 \_3$ & 5x5_1 & $5 \times 5 \_2$ & 5x5_3 & $6 \times 6 \_1$ & $6 x 6 \_2$ & $6 \times 6 \_3$ & 7x7_1 & $7 \times 7 \_2$ & $7 \times 7 \_3$ \\
\hline
\end{tabular}

Table 2 - Differential throughput (EIED, BEB) 


\begin{tabular}{|c|c|c|c|c|c|c|c|c|c|c|c|c|c|c|c|}
\hline 400 & -29.69 & -14.60 & -18.65 & -87.69 & 23.74 & -5.18 & -193.12 & -181.81 & -11.61 & -516.65 & -152.04 & -237.34 & -800.60 & -483.97 & -267.89 \\
\hline 600 & -10.58 & -3.77 & -24.50 & -8.29 & -32.40 & -18.16 & -104.02 & -54.95 & -34.12 & -372.03 & -211.94 & -180.11 & -775.10 & -509.04 & -261.62 \\
\hline 800 & -34.40 & -4.97 & 2.63 & -54.66 & -46.15 & 24.17 & -267.23 & 66.46 & 51.70 & -344.52 & -143.03 & -34.25 & -565.19 & -248.74 & -119.74 \\
\hline 1000 & -0.44 & 7.73 & -8.23 & 38.38 & -68.76 & -100.19 & 30.35 & 62.63 & -45.30 & -287.44 & -30.84 & 8.78 & -322.98 & -62.94 & -42.53 \\
\hline 1200 & 3.65 & 16.16 & -4.36 & -13.83 & 55.20 & -27.21 & -126.65 & -63.53 & -33.77 & -195.45 & -18.28 & 23.87 & -600.19 & -254.18 & -115.23 \\
\hline 1400 & 3.88 & 2.93 & -6.51 & 24.47 & -94.75 & -6.97 & 78.18 & -38.58 & 24.54 & 1.30 & -66.36 & -121.51 & -218.78 & -83.28 & -32.49 \\
\hline 1500 & 15.71 & 9.32 & 19.28 & 18.41 & -46.16 & 3.03 & -63.93 & 44.54 & -4.46 & -252.76 & 12.67 & -60.20 & -260.90 & 98.93 & -206.56 \\
\hline 1600 & 31.15 & 7.22 & 21.08 & 11.87 & 12.91 & 8.23 & -57.88 & 18.35 & -45.82 & -222.84 & 26.12 & -74.58 & -209.60 & 24.87 & -6.85 \\
\hline 1800 & -30.77 & -14.04 & -2.74 & -64.05 & 28.84 & 37.89 & -130.60 & 117.61 & 104.74 & -242.35 & 232.48 & 44.18 & -234.52 & -37.89 & -26.85 \\
\hline 2000 & -7.19 & 8.16 & -5.87 & 67.65 & -0.06 & -45.61 & -21.32 & 112.20 & 85.82 & 31.98 & 166.14 & -26.25 & -23.06 & -200.33 & 162.56 \\
\hline
\end{tabular}

Inference-1: It can be inferred from Table 2 that EIED with packet sizes 1200 to 2000 bytes performs better with lesser radio interfaces when compared with BEB. (Refer. Columns 3x3_p - 5x5_p, where p=1/2/3)

Inference-2: With highly dense networks the performance is always observed to be to poorer when compared with BEB (Refer columns 7x7_1, 7x7_2, 7x7_3)

Table 3- Differential throughput (DIDD, BEB)

\begin{tabular}{|c|c|c|c|c|c|c|c|c|c|c|c|c|c|c|c|}
\hline \multicolumn{16}{|c|}{ THDIDD - THBEB } \\
\hline SIZE & 3x3_1 & 3x3_2 & 3x3_3 & $4 \times 4 \_1$ & $4 \times 4 \_2$ & $4 \times 4 \_3$ & $5 \times 5 \_1$ & $5 \times 5 \_2$ & $5 \times 5 \_3$ & 6x6_1 & $6 \times 6 \_2$ & 6x6_3 & 7x7_1 & $7 \times 7 \_2$ & 7x7_3 \\
\hline 400 & -5.71 & 16.62 & 3.23 & -70.69 & 57.14 & -13.60 & -108.93 & -62.11 & 68.10 & -286.34 & -43.45 & -61.92 & -417.39 & -81.31 & -3.02 \\
\hline 600 & -4.76 & -6.17 & -3.49 & 34.97 & -64.63 & 4.21 & 41.75 & -20.53 & -20.46 & -248.90 & -140.46 & -122.09 & -345.72 & -281.50 & -209.35 \\
\hline 800 & -18.06 & 7.66 & 4.92 & -33.97 & -9.96 & 40.69 & -128.94 & 49.23 & 81.73 & -174.01 & -68.85 & 33.98 & -184.82 & -59.00 & 193.75 \\
\hline 1000 & -7.15 & 4.51 & -13.18 & -16.11 & -98.19 & -35.85 & 85.78 & -9.32 & -36.46 & -39.54 & 37.02 & 117.47 & -22.93 & -109.51 & -75.89 \\
\hline 1200 & -2.96 & 12.75 & 9.35 & -35.78 & 20.40 & 28.09 & -27.24 & -73.16 & -76.89 & 8.95 & 57.88 & 144.06 & -268.19 & -119.84 & 8.46 \\
\hline 1400 & 1.97 & 4.70 & -6.48 & 4.07 & 7.03 & -29.65 & 60.16 & -46.26 & -51.65 & 33.69 & -35.11 & -202.91 & -96.83 & 90.44 & -0.27 \\
\hline 1500 & 24.33 & 9.39 & 7.89 & 28.73 & -51.19 & -15.34 & -24.33 & -1.24 & 26.31 & -117.47 & 192.24 & 12.98 & -259.83 & 152.70 & -157.67 \\
\hline 1600 & 10.61 & 0.60 & -7.66 & -9.93 & 111.90 & -17.82 & -61.38 & -34.60 & -29.19 & -53.55 & 5.49 & 79.25 & 13.57 & 217.26 & 242.06 \\
\hline 1800 & -29.66 & -0.17 & -2.21 & -72.55 & 15.61 & 81.44 & -81.90 & 7.17 & 87.77 & -114.43 & 336.08 & 60.02 & -123.38 & 55.83 & -6.77 \\
\hline 2000 & -15.17 & 6.22 & -11.93 & 6.91 & -64.16 & -47.92 & -76.35 & 54.65 & 21.31 & 2.47 & 47.98 & -157.46 & 159.86 & 113.19 & 158.85 \\
\hline
\end{tabular}

Inference-1: It is suggested to use DIDD with packet sizes between 1200 and 1800 bytes with TWO or THREE number of interfaces. But with single interface, it performs poorly when compared to BEB. Refer Columns AAxAA_1 (where AA = 3, 4, $5,6,7)$

Inference-2: DIDD perform better than BEB with THREE radio interfaces irrespective of the size of packets, whereas with ONE / TWO radios, it is suggested to operate with packet sizes between 1200 and 2000 bytes.

Table 4 - Differential throughput (MILD, BEB)

\begin{tabular}{|c|c|c|c|c|c|c|c|c|c|c|c|c|c|c|c|}
\hline \multicolumn{16}{|c|}{ THMILd - THвEB } \\
\hline SIZE & 3x3_1 & 3x3_2 & 3x3_3 & 4x4_1 & $4 \times 4 \_2$ & $4 \times 4 \_3$ & 5x5_1 & $5 \times 5 \_2$ & $5 \times 5 \_3$ & 6x6_1 & 6x6_2 & $6 \times 6 \_3$ & 7x7_1 & $7 \times 7 \_2$ & 7x7_3 \\
\hline 400 & -66.94 & -55.71 & -72.42 & -270.82 & -97.12 & -11.80 & -496.73 & -345.71 & -112.10 & -879.08 & -587.82 & -687.56 & -1400.16 & -1100.32 & -1101.29 \\
\hline 600 & -58.71 & -16.18 & -81.96 & -162.34 & -97.96 & -2.91 & -414.22 & -97.80 & -216.30 & -906.18 & -633.84 & -555.11 & -1362.04 & -1061.81 & -794.82 \\
\hline 800 & -51.55 & -44.21 & -16.29 & -134.60 & -84.89 & -26.63 & -408.99 & -60.04 & -85.81 & -769.91 & -528.53 & -392.28 & -985.77 & -795.70 & -443.05 \\
\hline 1000 & -18.16 & -20.07 & -13.07 & -50.73 & -102.04 & -123.10 & -49.10 & -38.57 & -126.57 & -499.26 & -314.36 & -164.73 & -923.78 & -791.78 & -434.61 \\
\hline 1200 & 24.77 & 13.85 & -3.50 & -52.56 & -15.56 & 27.93 & -287.85 & -205.33 & -148.85 & -451.39 & -119.79 & -254.52 & -965.72 & -504.80 & -391.17 \\
\hline 1400 & -10.04 & 7.55 & 0.33 & -10.16 & -75.02 & -29.62 & -7.77 & -71.56 & -116.19 & -227.86 & -195.88 & -310.46 & -594.07 & -499.84 & -430.97 \\
\hline 1500 & 17.18 & 4.79 & 16.23 & -18.38 & -94.41 & 28.25 & -160.70 & 17.71 & -64.51 & -424.92 & -30.84 & -194.15 & -581.85 & -200.78 & -488.82 \\
\hline 1600 & 21.41 & 6.06 & 10.58 & -23.26 & 24.18 & -1.84 & -81.95 & -133.06 & -70.56 & -525.31 & -285.19 & -341.38 & -393.18 & -177.28 & -535.21 \\
\hline 1800 & -16.67 & 14.04 & 0.88 & -108.35 & -46.27 & 56.80 & -75.67 & 62.60 & 28.13 & -188.53 & 83.54 & -187.79 & -678.63 & -250.06 & -487.02 \\
\hline 2000 & 4.07 & 11.79 & -0.40 & -3.56 & -45.84 & -25.98 & -53.69 & 83.64 & 108.86 & -335.59 & 55.43 & -177.17 & -235.06 & -428.68 & -119.06 \\
\hline
\end{tabular}




\section{Performance Characterization of IEEE 802.11s Compatible MAC Protocols}

Inference-1: It can be inferred that MILD perform very poorly when compared to BEB with smaller sized packets (less than $1.2 \mathrm{~KB})$.

Inference-2: It is suited for lightly dense networks (3x3_1, 3x3_2, 3x3_3) with packet size between 1200 and 2000 bytes.

Table 5 - Differential throughput (EILD_32, BEB)

\begin{tabular}{|c|c|c|c|c|c|c|c|c|c|c|c|c|c|c|c|}
\hline \multicolumn{16}{|c|}{ THEILD $32-$ TH$_{\text {BEB }}$} \\
\hline SIZE & 3x3_1 & 3x3_2 & 3x3_3 & $4 \times 4 \_1$ & $4 \times 4 \_2$ & $4 \times 4 \_3$ & 5x5_1 & 5x5_2 & $5 \times 5 \_3$ & 6x6_1 & 6x6_2 & 6x6_3 & 7x7_1 & 7x7_2 & 7x7_3 \\
\hline 400 & -118.62 & -59.30 & -107.34 & -263.31 & -102.30 & -146.14 & -712.05 & -519.12 & -212.63 & -1125.33 & -957.55 & -712.09 & -1583.63 & -1543.52 & -1251.82 \\
\hline 600 & -72.38 & -67.31 & -85.35 & -258.91 & -124.15 & -90.44 & -488.74 & -283.56 & -151.12 & -1022.09 & -745.18 & -535.47 & -1527.99 & -1170.90 & -919.99 \\
\hline 800 & -76.44 & -12.01 & -40.10 & -160.11 & -54.95 & -44.51 & -448.84 & -64.34 & -143.50 & -802.80 & -501.80 & -405.79 & -1302.13 & -839.03 & -579.35 \\
\hline 1000 & -28.25 & -2.48 & -28.52 & -65.38 & -121.78 & -107.98 & -170.76 & -104.49 & -197.80 & -654.47 & -287.54 & -264.66 & -1103.00 & -729.16 & -629.36 \\
\hline 1200 & -4.42 & 14.07 & 1.85 & -92.79 & 8.09 & -15.00 & -291.36 & -169.66 & -53.99 & -490.78 & -243.77 & -297.88 & -1071.01 & -684.15 & -456.11 \\
\hline 1400 & -12.53 & 16.54 & 3.73 & -38.27 & -114.64 & -24.50 & -38.12 & -56.00 & -80.00 & -262.24 & -258.68 & -348.32 & -663.85 & -508.70 & -557.97 \\
\hline 1500 & 0.45 & 18.73 & 6.46 & -15.14 & -55.61 & 12.09 & -190.13 & 44.82 & -90.76 & -557.63 & -133.96 & -335.37 & -678.15 & -235.43 & -504.58 \\
\hline 1600 & 5.92 & 10.61 & 19.74 & -54.17 & 19.23 & 10.07 & -124.95 & -93.47 & -60.10 & -361.75 & -138.32 & -180.74 & -570.01 & -287.97 & -383.23 \\
\hline 1800 & -19.49 & 19.32 & 17.78 & -102.76 & 60.36 & 87.05 & -233.84 & 24.84 & 94.03 & -387.07 & 157.39 & -87.44 & -598.90 & -156.66 & -439.98 \\
\hline 2000 & 3.30 & 15.54 & 8.52 & 36.69 & -17.10 & 17.72 & -165.82 & 29.26 & 58.78 & -275.85 & 54.70 & -5.65 & -418.26 & -501.05 & -47.28 \\
\hline
\end{tabular}

Inference-1: It can be inferred that EILD_32 perform very poorly when compared to BEB with smaller sized packets (less than $1.2 \mathrm{~KB})$.

Inference-2: It is suited for lightly dense networks with packet size between 1200 and 2000 bytes.

Table 6 - Differential throughput (EILD_64, BEB)

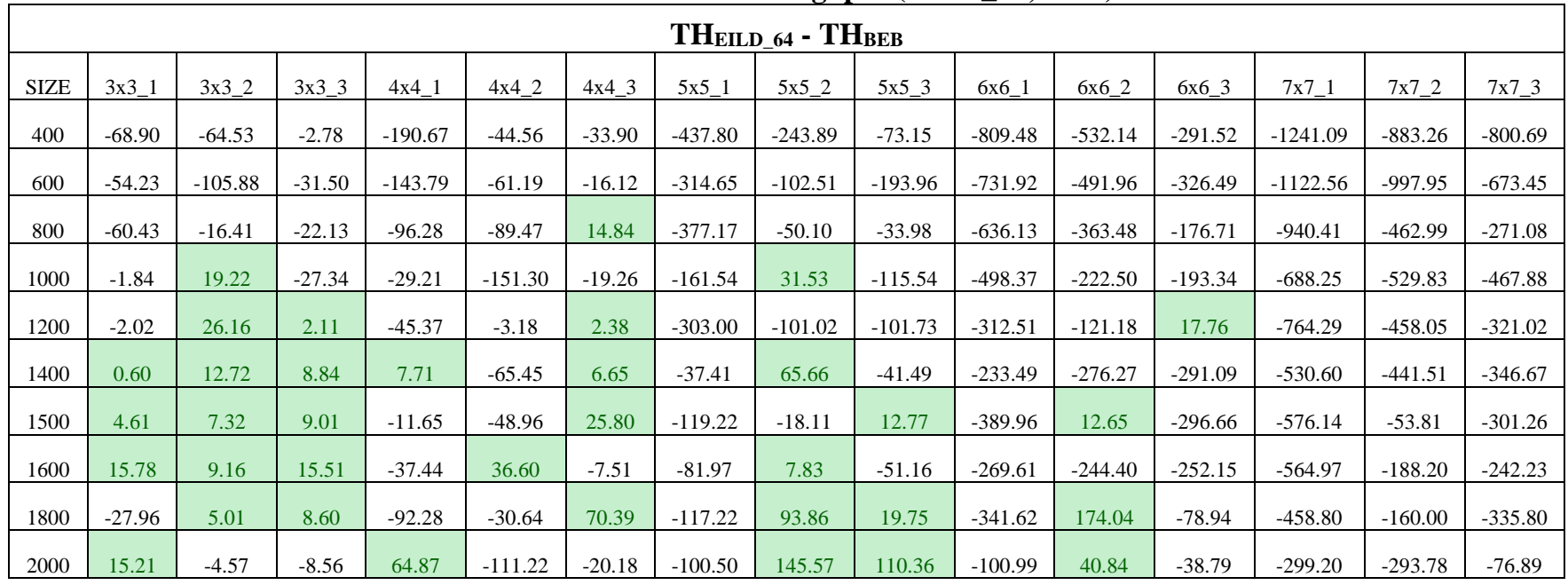

Inference-1: It can be inferred that EILD_64 perform very poorly when compared to BEB with smaller sized packets (Rows1-4 $-400,600,800$, and 1000).

Inference-2: It is suited for lightly dense networks with packet size between $1.2 \mathrm{~KB}$ and $2.0 \mathrm{~KB}$.

Inference-3: It can be inferred from Table 5 -

Table 6 that EILD_64 works better than EILD_32.

Table 7- - Differential throughput (DCF_CCW_300, BEB)

\begin{tabular}{|c|c|c|c|c|c|c|c|c|c|c|c|c|c|c|c|}
\hline \multicolumn{16}{|c|}{ THDCF_CCW_300 - THBEB } \\
\hline SIZE & 3x3_1 & 3x3_2 & 3x3_3 & 4x4_1 & $4 \times 4 \_2$ & $4 \times 4 \_3$ & 5x5_1 & $5 \times 5 \_2$ & 5x5_3 & 6x6_1 & $6 \times 6 \_2$ & 6x6_3 & 7x7_1 & 7x7_2 & 7x7_3 \\
\hline 400 & -50.52 & -39.83 & -42.43 & -136.22 & -47.11 & -65.71 & -266.16 & -214.82 & -116.97 & -495.04 & -268.01 & -344.48 & -666.89 & -604.25 & -648.04 \\
\hline 600 & -45.74 & -30.13 & -30.77 & -61.04 & -79.69 & -26.91 & -36.16 & -41.49 & -120.82 & -284.53 & -312.34 & -231.41 & -558.10 & -398.94 & -324.80 \\
\hline 800 & -8.88 & -14.87 & -5.92 & -34.70 & -42.09 & -23.49 & -76.25 & 18.90 & -1.79 & -263.23 & -123.15 & -212.76 & -339.37 & -294.31 & 80.74 \\
\hline
\end{tabular}

Published By:

Blue Eyes Intelligence Engineering 


\begin{tabular}{|c|c|c|c|c|c|c|c|c|c|c|c|c|c|c|c|}
\hline 1000 & 3.10 & -5.37 & -11.06 & 11.33 & -21.73 & -89.43 & 59.26 & -82.38 & -56.78 & -74.88 & -86.64 & 1.84 & -24.52 & -164.08 & -90.27 \\
\hline 1200 & 7.52 & 4.66 & 3.22 & -27.19 & 23.14 & -10.89 & -108.54 & -115.79 & -165.80 & -2.35 & -1.85 & -58.39 & -264.24 & -199.98 & -150.89 \\
\hline 1400 & 3.21 & -1.45 & -6.23 & 24.41 & -126.78 & -36.81 & 137.67 & -76.17 & -143.27 & 32.72 & -97.44 & -307.90 & 1.14 & -43.02 & -195.58 \\
\hline 1500 & 13.09 & 1.79 & 11.52 & 47.01 & -68.52 & -49.21 & -95.49 & -17.61 & -34.41 & -106.94 & 5.08 & -278.21 & -27.25 & -168.21 & -280.62 \\
\hline 1600 & 19.44 & -3.91 & 16.96 & 18.18 & 91.65 & 0.17 & -22.06 & -117.34 & -113.55 & -14.71 & -132.42 & -220.51 & -46.08 & 105.22 & -97.90 \\
\hline 1800 & -31.39 & -5.78 & 0.23 & -64.72 & 45.35 & 54.21 & -100.57 & 47.28 & 8.69 & -141.12 & 177.42 & -187.01 & -84.85 & -64.84 & -144.67 \\
\hline 2000 & 2.13 & 2.18 & -1.03 & 34.03 & -74.94 & -49.97 & -124.21 & -30.44 & -38.37 & 57.02 & 43.67 & -99.54 & 165.82 & -263.14 & 107.85 \\
\hline
\end{tabular}

Inference-1: It can be inferred that DCF with CCW (cw=300) mostly underperform with packets less than $1 \mathrm{~KB}$. Otherwise it works better than $\mathrm{BEB}$ for most of the network configurations.

Inference-2: It can be inferred that DCF with CCW $(\mathrm{cw}=300)$ mostly works better with lesser dense networks.

Table 8- Differential throughput (DCF CCW (w=400), BEB)

\begin{tabular}{|c|c|c|c|c|c|c|c|c|c|c|c|c|c|c|c|}
\hline \multicolumn{16}{|c|}{ THDCF_CCW_400 - THBEB } \\
\hline SIZE & 3x3_1 & $3 \times 3 \_2$ & $3 \times 3 \_3$ & $4 \times 4 \_1$ & $4 \times 4 \_2$ & $4 \times 4 \_3$ & $5 \times 5 \_1$ & $5 \times 5 \_2$ & 5x5_3 & 6x6_1 & 6x6_2 & 6x6_3 & 7x7_1 & $7 \times 7 \_2$ & 7x7_3 \\
\hline 400 & -62.49 & -81.51 & -63.73 & -213.28 & -139.04 & -117.60 & -349.42 & -315.04 & -201.86 & -637.89 & -464.17 & -511.31 & -1070.51 & -885.90 & -748.67 \\
\hline 600 & -37.13 & -70.15 & -48.13 & -91.24 & -75.72 & -49.31 & -166.58 & -68.08 & -124.83 & -455.66 & -259.20 & -226.93 & -582.77 & -589.74 & -295.28 \\
\hline 800 & -36.75 & -22.97 & -7.58 & -79.11 & -67.39 & -25.00 & -175.20 & 13.73 & -29.05 & -225.52 & -109.56 & -109.07 & -340.73 & -264.77 & -70.91 \\
\hline 1200 & -11.85 & 11.36 & 0.13 & -31.39 & -21.69 & 4.60 & -115.33 & -157.70 & -77.26 & -140.11 & -101.67 & -106.84 & -118.95 & -161.36 & -10.50 \\
\hline 1400 & 7.40 & -4.24 & -15.80 & -2.84 & -81.27 & -30.64 & 102.12 & -1.17 & 11.60 & -1.35 & -166.69 & -221.88 & -9.79 & 94.45 & -85.92 \\
\hline 1500 & 12.92 & -2.05 & 1.74 & -7.21 & -27.00 & 40.86 & 24.94 & 53.54 & -33.83 & -83.61 & 73.37 & -129.23 & -38.64 & 109.79 & -273.20 \\
\hline 1600 & 17.97 & -11.20 & 24.71 & 3.33 & 138.74 & -33.36 & 14.50 & -19.39 & -81.21 & -70.53 & -127.95 & -38.10 & 60.72 & 18.78 & 4.46 \\
\hline 1800 & 11.16 & 17.45 & -4.92 & -33.25 & -40.52 & 58.33 & -10.57 & 50.47 & 50.16 & -118.98 & 156.55 & 11.12 & -129.21 & 258.03 & -118.81 \\
\hline 2000 & 32.44 & 6.27 & -19.61 & 20.54 & -39.48 & -73.14 & -60.87 & 73.49 & 49.60 & 62.52 & 36.32 & -130.81 & 244.72 & -121.05 & 262.53 \\
\hline
\end{tabular}

Inference-1: It can be inferred that DCF with CCW (cw=400) mostly underperform with packets less than 1.2KB. Otherwise it works better than BEB most of the network configurations.

Inference-2: It can be inferred that DCF with CCW $(\mathrm{cw}=400)$ mostly works better with lesser dense networks.

Table 9 - Differential throughput (PB_PAPER_2, BEB)

\begin{tabular}{|c|c|c|c|c|c|c|c|c|c|c|c|c|c|c|c|}
\hline \multicolumn{16}{|c|}{ THPB_PAPER_2 - TH } \\
\hline SIZE & 3x3_1 & $3 \times 3 \_2$ & 3x3_3 & $4 \times 4 \_1$ & $4 \times 4 \_2$ & $4 \times 4 \_3$ & 5x5_1 & $5 \times 5 \_2$ & $5 \times 5 \_3$ & 6x6_1 & $6 \times 6 \_2$ & 6x6_3 & 7x7_1 & $7 \times 7 \_2$ & $7 x 7 \_3$ \\
\hline 400 & 5.15 & 8.74 & 5.85 & 48.03 & 25.78 & 17.44 & 18.41 & -79.13 & 131.58 & -73.10 & -72.46 & -32.57 & -175.87 & -77.20 & 7.83 \\
\hline 600 & -0.43 & -8.33 & -9.37 & 23.35 & -15.05 & 28.18 & 51.42 & -27.70 & 22.49 & -67.97 & -71.70 & -45.71 & 5.84 & -169.71 & -37.35 \\
\hline 800 & -21.84 & 4.99 & 1.93 & -50.58 & -39.70 & 52.08 & -7.12 & 67.95 & -18.67 & -151.74 & -51.56 & 47.08 & -156.04 & 95.70 & 117.55 \\
\hline 1000 & 0.53 & -2.16 & -4.18 & 31.30 & -86.32 & -35.50 & 113.41 & 16.81 & 72.07 & -62.58 & 68.70 & 87.82 & 98.53 & 99.31 & 94.07 \\
\hline 1200 & -1.27 & 11.04 & -0.60 & 9.81 & 20.40 & 23.43 & -85.91 & -101.41 & 3.67 & 118.16 & 105.46 & -17.47 & -432.38 & -108.66 & -29.81 \\
\hline 1400 & 46.53 & 1.27 & 9.29 & 5.44 & -93.56 & 0.83 & 211.40 & 16.86 & -27.30 & 91.28 & -141.39 & -196.96 & -103.04 & 52.62 & -114.93 \\
\hline 1500 & 13.29 & -4.85 & 8.91 & 37.70 & 21.29 & 9.23 & 6.21 & 35.33 & -30.64 & -51.74 & 189.76 & -52.32 & -24.51 & 157.03 & -278.52 \\
\hline 1600 & 26.15 & -7.43 & 2.85 & 17.39 & 51.08 & -82.87 & -68.83 & -8.39 & 38.38 & -73.09 & 62.62 & -107.01 & 62.50 & 101.78 & 137.15 \\
\hline 1800 & -44.48 & 6.05 & -3.77 & -96.72 & -54.41 & 58.08 & -59.32 & -35.48 & 102.65 & -159.47 & 199.10 & -130.45 & -127.78 & 154.18 & -229.68 \\
\hline 2000 & -1.33 & 8.87 & -5.16 & 33.56 & -46.42 & -33.04 & -60.44 & 37.82 & 42.49 & 23.03 & 140.42 & 78.50 & 198.30 & -218.59 & 111.18 \\
\hline
\end{tabular}

Inference-1: It can be inferred that Polynomial backoff (PB) works well when compared to BEB, Irrespective of number of interfaces, node density, packet sizes.

Inference-2: It can be inferred that with 3 radio interfaces (3x3_3, 4x4_3, 5x5_3, 6x6_3. 7x7_3), PB works better than BEB.

Table 10 - Differential throughput (EBO, BEB)

Retrieval Number: C5693029320/2020@BEIESP

DOI: 10.35940/ijeat.C5693.029320

Journal Website: www.ijeat.org 


\section{Performance Characterization of IEEE 802.11s Compatible MAC Protocols}

\begin{tabular}{|c|c|c|c|c|c|c|c|c|c|c|c|c|c|c|c|}
\hline \multicolumn{16}{|c|}{ THebo - THвев } \\
\hline SIZE & $3 \times 3 \_1$ & $3 \times 3 \_2$ & $3 \times 3 \_3$ & $4 \times 4 \_1$ & $4 \times 4 \_2$ & $4 \times 4 \_3$ & $5 \times 5 \_1$ & $5 \times 5 \_2$ & $5 \times 5 \_3$ & $6 \times 6 \_1$ & $6 \times 6 \_2$ & $6 \times 6 \_3$ & $7 \times 7 \_1$ & $7 \times 7 \_2$ & $7 \times 7 \_3$ \\
\hline 400 & 11.03 & 30.26 & 7.08 & -96.40 & 8.40 & -10.30 & -281.37 & -60.96 & 42.54 & -514.13 & -17.69 & -208.49 & -630.07 & -272.59 & -263.31 \\
\hline 600 & -1.41 & -31.22 & -32.57 & -51.46 & -16.66 & 20.47 & -181.10 & -26.88 & -18.19 & -403.21 & -103.53 & -128.85 & -709.32 & -378.73 & -281.96 \\
\hline 800 & -13.72 & 11.83 & 3.83 & 1.35 & -78.16 & -7.79 & -161.16 & 107.11 & 1.82 & -291.20 & -163.00 & -90.96 & -310.10 & -34.09 & -45.54 \\
\hline 1000 & 2.34 & 1.41 & -20.14 & 4.10 & -11.77 & -31.60 & -35.51 & 71.81 & -46.04 & -227.11 & -5.27 & -41.32 & -365.09 & -193.49 & -250.05 \\
\hline 1200 & -2.75 & 9.10 & -18.08 & -43.89 & 63.60 & 8.28 & -82.78 & -102.71 & -47.64 & -108.52 & 99.75 & 1.66 & -474.30 & -190.01 & 240.29 \\
\hline 1400 & 1.16 & 9.41 & 2.48 & -8.89 & -52.15 & -17.94 & 18.72 & -48.48 & -56.89 & -10.20 & -104.00 & -107.44 & -167.86 & -167.16 & -127.14 \\
\hline 1500 & 14.26 & -10.85 & 1.40 & 18.23 & -92.86 & 26.70 & 8.92 & 56.63 & -75.76 & 28.31 & 27.06 & -10.03 & -195.44 & 102.10 & -53.48 \\
\hline 1600 & 16.93 & 11.72 & 11.61 & 10.77 & -29.89 & 5.30 & -60.64 & -48.02 & -95.82 & -181.24 & -1.67 & -159.00 & -192.15 & 8.17 & -95.35 \\
\hline 1800 & -29.68 & 2.73 & 5.73 & -100.40 & 80.26 & 67.57 & -119.12 & 41.52 & 93.13 & -231.84 & 155.88 & -107.47 & -172.75 & -20.00 & 19.47 \\
\hline 2000 & 12.02 & 4.67 & -6.48 & 29.20 & -150.26 & -8.27 & -131.73 & 85.00 & 17.18 & -38.76 & 28.49 & -107.88 & -1.33 & 15.57 & 339.56 \\
\hline
\end{tabular}

Inference-1: It is observed that EBO works well with small network sizes irrespective of number of interfaces supported by the Mesh router (Refer Columns -

Table 10 , 3x3_1, 4x4_1, 3x3_2, 4x4_2, 3x3_3, 4x4_3)

Inference-2: With larger network sizes irrespective of number of interfaces supported by the Mesh router the performance is not better than BEB (Refer Columns -

Table 10 ,5x5_q, 6x6_q, 7x7_q, where q=1, 2, 3)

Table 11 - Differential throughput (HBO, BEB)

\begin{tabular}{|c|c|c|c|c|c|c|c|c|c|c|c|c|c|c|c|}
\hline \multicolumn{16}{|c|}{ ТНнво - ТНвев } \\
\hline SIZE & 3x3_1 & 3x3_2 & 3x3_3 & $4 \times 4 \_1$ & $4 \times 4 \_2$ & $4 \times 4$ _3 & 5x5_1 & $5 \times 5 \_2$ & $5 \times 5 \_3$ & 6x6_1 & 6x6_2 & 6x6_3 & 7x7_1 & 7x7_2 & 7x7_3 \\
\hline 400 & 10.22 & -2.59 & 16.43 & 46.29 & 62.34 & -27.51 & -6.92 & -82.10 & 48.05 & 93.03 & -37.16 & 87.07 & -131.90 & -15.73 & 12.44 \\
\hline 600 & 7.49 & -12.18 & -11.74 & -38.12 & -41.82 & -3.94 & 74.31 & -39.29 & -47.24 & -20.70 & -60.05 & -105.37 & -74.66 & -134.91 & -142.67 \\
\hline 800 & -10.01 & -5.02 & -5.80 & -20.72 & -26.45 & 6.99 & -24.99 & 31.23 & 69.34 & -46.06 & -42.65 & -88.71 & -184.94 & 51.76 & 302.60 \\
\hline 1000 & -4.51 & 8.78 & -4.91 & 5.71 & -117.92 & -65.48 & 100.30 & 0.50 & -58.15 & 11.60 & 29.63 & 87.80 & 18.28 & -14.18 & -40.04 \\
\hline 1200 & 0.29 & 19.16 & 1.59 & 0.18 & 160.08 & -48.90 & 11.10 & -58.28 & -87.18 & 13.38 & 96.46 & 25.67 & -207.87 & -163.26 & 0.17 \\
\hline 1400 & -1.69 & -4.25 & 2.57 & 2.51 & -45.61 & -17.42 & 67.14 & -71.40 & -2.07 & 51.96 & -115.36 & -264.72 & 92.90 & 231.45 & -28.39 \\
\hline 1500 & 9.13 & 4.60 & 0.57 & 75.92 & -9.97 & -21.89 & -62.06 & 43.23 & 60.04 & -142.90 & -39.44 & -38.19 & 72.11 & 11.83 & -148.93 \\
\hline 1600 & 15.56 & -12.52 & 11.10 & 57.04 & 58.30 & -33.33 & 36.09 & -71.88 & -5.37 & -218.02 & 50.59 & -98.66 & 65.33 & 254.60 & -123.16 \\
\hline 1800 & -32.73 & 2.84 & 5.82 & -69.59 & 6.16 & -4.99 & -77.81 & 15.73 & 6.10 & -89.60 & 142.82 & -6.15 & -179.85 & 1.56 & -297.42 \\
\hline 2000 & 1.40 & 2.35 & 0.26 & 2.96 & -22.82 & -84.54 & -122.91 & 35.00 & -32.58 & 176.32 & -145.15 & 17.43 & 251.59 & -240.97 & -50.06 \\
\hline
\end{tabular}

Inference-1: It is observed that HBO works well with majority of the scenarios but consistency is not guaranteed.

Inference-2: It is observed that HBO with THREE interfaces does not guarantee throughput enhancement (Refer Columns Table 11, ZxZ_3, where $\mathrm{Z}=4,6,7$ ).

\section{Overall inference:}

1. It is inferred that BEB algorithm may not be the best choice for mesh routers in WMNs.

2. It is inferred that the WMNs shall be operated with packet sizes in between $1 \mathrm{~KB}$ and $2 \mathrm{~KB}$ to achieve better throughput performance.

3. Considering Columns $\left(3 \times 3 \_3,4 x 4 \_3,5 \times 5 \_3,6 \times 6 \_3\right.$, 7x7_3) from Table 2 - Table 11, it can be inferred that PB is the best choice to obtain higher throughput.

4. For a given router, MAC protocol implementation cannot be changed at run time depending upon the node density. Thus a MAC protocol which works well with almost all the network configurations shall be selected for a given Mesh router implementation. Among various MAC protocols considered in this work, polynomial backoff (PB) algorithm appears to be a better choice for WMN installations. [24]

\section{CONCLUSION}

In this paper, we have presented the NS3 simulation results with BEB and its enhanced variant of CA algorithms (EIED, EILD, MILD, PB, CCW, $E B O, H B A \ldots)$ to evaluate their suitability for WMNs. 
The performance has been compared and contrasted in terms of packet size, grid size, number of radio interfaces. It is observed that polynomial backoff (PB) algorithm appears to be a better choice for WMNs instead of de-facto MAC CA algorithm (BEB) with few limitations.

Next alternate choices for WMN performance enhancement are EBO, HBO, EIED, and/or DIDD algorithms. Also enabling TWO radios on each of the mesh router helps to achieve better throughput performance.

Thus in future we plan to enhance polynomial backoff algorithm or combination of these algorithms to support higher throughput for WMNs.

\section{ACKNOWLEDGEMENTS}

I would like to convey my sincere thanks to Dept. of ECE, AIT, which supported in running the NS3 [3] simulations in order to obtain these results.

\section{REFERENCES}

1. I. F. Akyildiz, X. Wang and W. Wang, "Wireless mesh networks: a survey," Computer Networks, vol. 47, pp. 445-487, 2005.

2. G. R. Hiertz, D. Denteneer, S. Max, R. Taori, J. Cardona, L. Berlemann and B. Walke, "IEEE 802.11s: The WLAN Mesh Standard," IEEE Wireless Communications, vol. 17, pp. 104-111, 22010.

3. S. F. Tom Henderson and S. Roy, NS3-Simulator, 2006.

4. W. C. Anderton and M. Young, "Is Our Model for Contention Resolution Wrong? Confronting the Cost of Collisions," in Proceedings of the 29th ACM Symposium on Parallelism in Algorithms and Architectures, New York, NY, USA, 2017.

5. T. Imboden, K. Akkaya and Z. Moore, "Performance evaluation of wireless mesh networks using IEEE 802.11s and IEEE 802.11n," in 2012 IEEE International Conference on Communications (ICC), 2012.

6. D. Xu, T. Sakurai and H. L. Vu, "An Analysis of Different Backoff Functions for an IEEE 802.11 WLAN," in 2008 IEEE 68th Vehicular Technology Conference, 2008.

7. J. Sartthong, "Half binary exponential increment double decrement back-off algorithm to enhance the saturated throughput of IEEE802.11 wireless LAN," Journal of Thai Interdisciplinary research, vol. 14, pp. 21-28, 2019.

8. N.-O. Song, B.-J. Kwak, J. Song and M. E. Miller, "Enhancement of IEEE 802.11 distributed coordination function with exponential increase exponential decrease backoff algorithm," in The 57th IEEE Semiannual Vehicular Technology Conference, 2003. VTC 2003-Spring., 2003.

9. P. Chatzimisios, V. Vitsas and A. C. Boucouvalas, "DIDD backoff scheme: An enhancement to IEEE 802.11 DCF under burst transmission errors," in 2006 IEEE Sarnoff Symposium, 2006.

10. J. Sartthong, S. Sittichivapak, A. Kaewpukdee and I. Boonpikum, "Binary Exponential Increment Half Decrement backoff algorithm for IEEE802.11 wireless LANs," in 2013 10th International Conference on Electrical Engineering/Electronics, Computer, Telecommunications and Information Technology, 2013.

11. Z. J. Haas and J. Deng, "On optimizing the backoff interval for random access schemes," IEEE Transactions on Communications, vol. 51, pp. 2081-2090, 122003.

12. A. B. Nataraju, H. D. Maheshappa and B. R. Shilpashree, "Implementation and Performance Analysis of DCWA MAC Protocol for Multihop Wireless Networks," in International Journal of Science and Applied Information Technology (IJSAIT), 2015.

13. T. Sakurai and H. L. Vu, "MAC Access Delay of IEEE 802.11 DCF," IEEE Transactions on Wireless Communications, vol. 6, pp. 1702-1710, 52007.

14. B. Nithya, U. Gupta and H. Subbiah, "Optimizing medium access using integer sequences in wireless networks," in 2017 International Conference on Communication and Signal Processing (ICCSP), 2017.

15. S. Sridhar, J. Guo and S. Jha, "Channel assignment in multi-radio wireless mesh networks : A graph-theoretic approach," pp. 1-10, 12009.

16. X. Sun and L. Dai, "Backoff Design for IEEE 802.11 DCF Networks: Fundamental Tradeoff and Design Criterion," IEEE/ACM Transactions on Networking, vol. 23, pp. 300-316, 22015.
17. S. Bi and Y. J. Zhang, "Mitigating power law delays: The use of polynomial backoff in IEEE 802.11 DCF," in 2012 IEEE International Conference on Communications (ICC), 2012.

18. A. Balador, A. Movaghar and S. Jabbehdari, "History Based Contention Window Control in IEEE 802.11 MAC Protocol in Error Prone Channel," Journal of Computer Science, vol. 6, 12010.

19. R. Wang, J. Zhang and X. Zou, "Performance Analysis and Optimization of IEEE 802.11 DCF with Constant Contention Window," in 2008 ISECS International Colloquium on Computing, Communication, Control, and Management, 2008.

20. X. Peng, L. Jiang and G. Xu, "Performance Analysis of Hybrid Backoff Algorithm of Wireless LAN," in 2007 International Conference on Wireless Communications, Networking and Mobile Computing, 2007.

21. A. B. Nataraju, H. D. Maheshappa and A. Devkatte, "Performance analysis of HWMP protocol for Wireless Mesh networks using NS3," in 2016 IEEE Region 10 Conference (TENCON), 2016.

22. V. Bharghavan, A. Demers, S. Shenker and L. Zhang, "MACAW: A Media Access Protocol for Wireless LAN's," SIGCOMM Comput. Commun. Rev., vol. 24, p. 212-225, 101994.

23. H. Qi, Z. Hu, X. Wen and Z. Lu, "An Enhanced MAC Backoff Algorithm for Heavy User Loaded WLANs," in 2017 IEEE Wireless Communications and Networking Conference (WCNC), 2017.

24. A. B. Nataraju, H. D. Maheshappa and A. Devkatte, "Performance analysis of HWMP protocol for Wireless Mesh networks using NS3," in 2016 IEEE Region 10 Conference (TENCON), 2016.

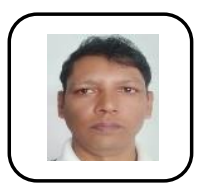

\section{AUTHORS’ PROFILE}

Mr. Nataraju A.B, Assistant Professor, Dept. of ECE AIT, Bangalore, completed his M.E degree from UVCE, Bangalore. He has work experience of over 9 years in Information Technology Industry (Huawei Technologies, Kodiak Networks, Sonus Networks,...) and over 10 years in academia. He is good with Computer Networking, VoIP, Internet Protocols, and Multimedia Communication. His research interest includes Wireless Networking, WMN performance enhancement, and wide area networking using WMNs. He has published about 10+ Conference/Journal publications. He is member of IEEE, IAENG, and CSI.

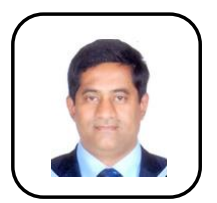

Dr. Hariprasad S.A, Director, School of Engineering and Technology, Jain University, Bangalore. He has work Experience of 25+ years (RVCE, BMSIT, DSU, ..) and guiding 12 research scholars. $3 \mathrm{PhD}$ scholars have been awarded with Ph.D. He is an expert in the field of Microcontrollers, Field theory, embedded systems. His Research Areas include Micro Controller, embedded system design, Machine learning etc., He has published more than 95 international Journal/Conference publications, and has got research fund of 5 Lakhs. 